\title{
Validation and refinement of expert systems: interpretation of NMR spectra as an application in analytical chemistry
}

\author{
R. Wehrens *, L. Buydens and G. Kateman \\ Laboratory for Analytical Chemistry, Faculty of Science, Catholic University, Toernooiveld, \\ 6525 ED Nijmegen (Netherlands)
}

(Received 11 September 1990; accepted 25 February 1991)

\begin{abstract}
Wehrens, R., Buydens, L. and Kateman, G., 1991. Validation and refinement of expert systems: interpretation of NMR spectra as an application in analytical chemistry. Chemometrics and Intelligent Laboratory Systems, 12: 57-67.

In this article we propose a strategy for the validation and improvement of expert systems in analytical chemistry. An application of this approach in two-dimensional NMR spectrum interpretation is shown, and the results indicate that significant performance improvements can be achieved in this domain.
\end{abstract}

\section{INTRODUCTION}

Expert systems have become increasingly important in the field of analytical chemistry and are applied in different fields of interest such as highperformance liquid chromatography (HPLC) [1] and others [2-4]. In the past few years expert system technology has matured and real-world applications have begun to appear. In view of increasing demands from regulatory offices and GLP norms a proper validation of expert system performance is necessary. This validation should, in the ideal case, consist of a short-term and a long-term component. The short-term component should make sure the expert system satisfies the original requirements of the experts, whereas the long-term component should continually validate the system during the field work so that the system remains up to date over a longer period of time. This long-term validation requires a kind of flexibility of the expert system that will allow gradual changes to be incorporated in a simple way. However, the question of the programmatic validation of expert systems has only been addressed in the artificial intelligence (AI) literature. In most expert system applications conventional software validation techniques are used for the validation and verification of expert system performance, and the specific difficulties related to expert system technology are largely neglected. Because expert system problem solving is essentially non-algorithmic in nature, each problem may require a different solution path, and in most cases there is no way to validate completeness and 
consistency of a knowledge base a priori. For example, in analytical chemistry there are numerous problems for which there are an infinite number of possible solutions, so that it is impossible to scan the whole problem space. Another source of problems lies in the knowledge acquisition phase; the transfer of knowledge of a human expert to a knowledge base is generally regarded as the bottleneck in expert system building. Methodologies [5] and knowledge acquisition tools ([6], and references therein) have been developed to overcome this problem. Despite these methodologies it appears that there are areas in which experts have difficulties in formulating their knowledge, which is essentially heuristic in nature, in an implementable form. This is especially the case when experts have to estimate uncertain quantities [7], and can result in erroneous knowledge bases and incorrect expert system performance. Several domain-independent validation techniques have been developed to detect logical errors in the domain knowledge of expert systems [8-10], but none of them have really addressed the kind of 'heuristic' errors in a knowledge base mentioned above. It seems that the validation of the knowledge of an expert system should also be checked with domain-dependent methods for it to be reliable. For expert systems in chemistry this means that chemical 'meta-knowledge' should be added to the system.

An approach that can be used to validate the knowledge base of an expert system is the so-called refinement strategy [11,12]. In this approach, the model of the knowledge is believed to be essentially correct, and the refinement strategy is therefore only meant to provide a 'fine-tuning' of the system. The results of the expert system are compared with a database of solved cases provided by the expert, and in case of incorrect results the knowledge base is checked for the rules that are responsible for them. Meta-knowledge is used to propose small refinements to those rules in order to improve performance. This was successfully implemented in the programs SEEK and its successor, SEEK2 [11,12]. These expert systems operate in the field of medical diagnosis. Since an appropriate model exists in most problem domains in analytical chemistry, we believe this approach is very suitable for the validation of expert systems operating in this field. In this article we show an application of the refinement approach in NMR spectrum interpretation. This is an example of a classification task that is typical for expert system problem solving in analytical chemistry. Other possibilities of this approach could be mentioned. The long-term validation mentioned above is easily integrated by allowing the expert system to be continually refined during the field work. Eventually, no further refinements will be found, provided that no changes in conditions and environment take place. If they do occur, the refinement approach provides a way to adapt the expert system to the new circumstances. Another possibility of this approach is a system that would allow the user to install his or her own preferences after several cases of 'conflicting opinions', or a system that could be adapted to another or a broader class of problems. The last application is an example of the possibilities of the refinement approach in a learning expert system.

In the next section a more detailed account will be given on the refinement strategy, and after that a brief description of the test expert system will be given.

\section{The refinement approach}

In most expert systems, heuristic knowledge is captured in so-called 'situation-action rules'. Such a rule consists of two parts: the premise part, which contains the conditions that must be satisfied for the rule to fire, and the conclusion part, which contains the actions that should be taken if the conditions are satisfied. Thus, if the situation at hand matches the conditions in the premises then the actions in the conclusions are carried out. As a (simple) example:

IF the polarity of ?compound is high THEN the solubility-in-water of ?compound is good.

The refinement approach provides a way for the fine-tuning of a set of rules in an expert system by comparing the results of the expert system with a database of known cases (the solutions of the 
human expert) to determine what results of the expert system are incorrect. The rules responsible for these misinterpreted cases are then traced, and refinements are considered, possibly correcting the errors. After applying one or more refinements, the expert system is consulted again, using the refined rule set, and the new results are evaluated. This cycle continues until no further improvements in performance are possible.

In general, two types of errors can be found: false negative results (hereafter: FN) are results the expert system failed to conclude, and false positive (FP) results are results that are concluded incorrectly by the expert system. FP results are caused by rules that fire too fast or with too great an impact. A way to correct this is to make the premises more difficult to satisfy, so that the rule succeeds in fewer cases. This can be done, for example, by adding another premise, raising thresholds in the premises, etc. Another way of correcting a rule responsible for an FP result is to reduce the strength of its conclusion(s), so that it may be overruled by another rule concluding the correct result. These refinements are called spe- cializations, since they narrow the range in which the rule can be applied or its effect.

The opposite of a specialization is a generalization; this refinement is feasible in case of FN results. Examples of generalization are: making premises easier to satisfy or deleting premises and increasing the effect of conclusions. The rule will then succeed more often or have a larger effect on the eventual outcome.

In general, the refinements applied should preserve the knowledge originally given by the expert, so far as possible. This conservative approach should guarantee that the rules continue to make sense after several rounds of training, and is a result of the assumption that the knowledge of the expert system is essentially correct.

\section{The refinement approach in classification tasks}

In a classification expert system, a normal strategy is first to collect support for all hypotheses, to combine the support in some way, and finally to accept or reject hypotheses on the basis of the combined support. In general, certain

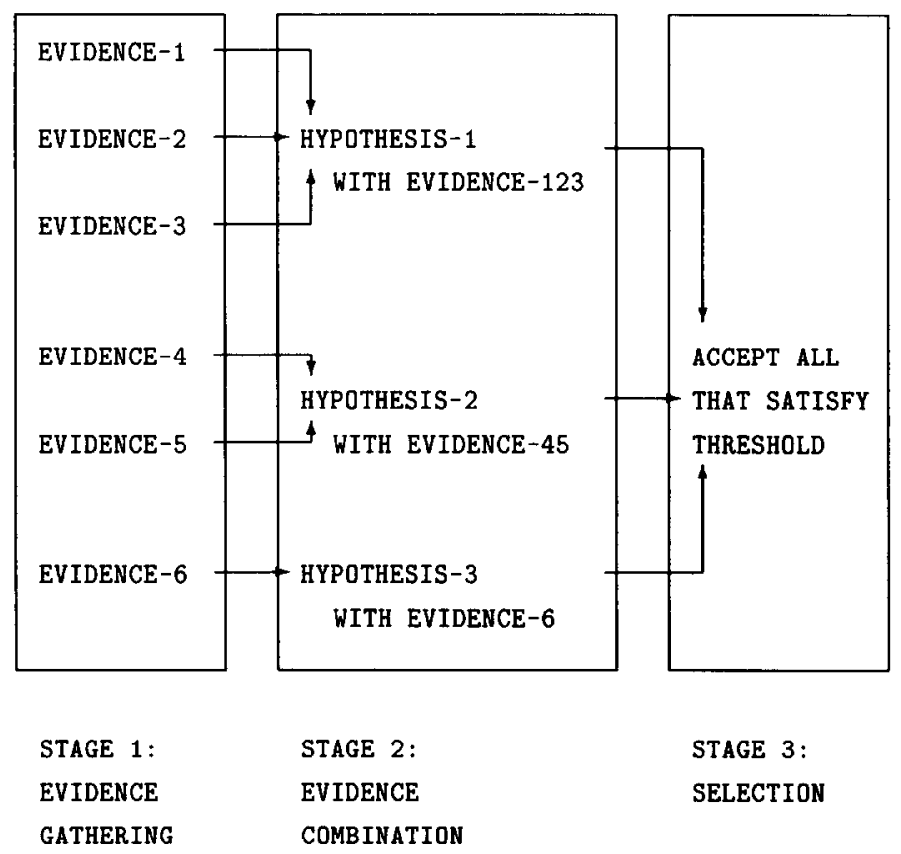

Fig. 1. The separate stages in a classification proces. At the left, the supports for the individual hypotheses, pictured in the middle box, are given. All hypotheses satisfying a certain criterion then are accepted (right box). 
thresholds must be satisfied to accept a hypothesis. These three stages are depicted in Fig. 1. In hierarchical classification, several schemes like the one in Fig. 1 are nested. In the second stage, the combination of evidence, no refinements are allowed because the knowledge in this stage is in fact control knowledge. On the numerical combination of support a vast amount of literature exists (see, e.g., refs. 13-15), and also symbolic types of support have been used [16]. The advantage of symbolic support is that experts typically have less difficulty in expressing their knowledge in linguistic, 'fuzzy' terms, such as 'big', 'small', 'major', etc., whereas estimating a numerical confidence value, for instance, often provides difficulties. Choosing a strategy to combine support largely depends on the knowledge representation and, because it falls somewhat beyond the scope of this article, we will not go into it any further.

At the level of the first and third stages refinements can be used to optimize the knowledge base; the importance of the individual items of evidence can be refined, and also thresholds that decide whether a hypothesis, based on its collected support, should be accepted or rejected. Allowing only a limited set of refinements will ensure that the proposed refinements will remain sensible from a chemical point of view. An additional advantage is the greater efficiency of a small set of refinements. During the knowledge acquisition stage it should be born in mind what parameters and what rules will be subject to refinements, so that no effort will have to be put in identifying the precise values for them in an early stage. It is, however, very important to note that the expert should always be the one who decides which of the proposed refinements is valid.

\section{TWO-DIMENSIONAL NMR OF PROTEINS}

In this section we briefly review the domain of the test expert system. Only those aspects that are necessary to an understanding of the results of the refinement approach will be discussed here. More information can be found in the references section and in the NMR literature. Two-dimensional ${ }^{1} \mathbf{H}$
NMR is now the most important technique for determining the structure of small and mediumsized proteins in solution. In such a spectrum the ordinary one-dimensional spectrum is more or less present on the diagonal, and the off-diagonal cross peaks give additional information, depending on the kind of spectrum. So called NOESY (Nuclear Overhauser Enhancement Spectroscopy) [17] spectra give information about the distance between protons: a cross peak between the two diagonal resonance positions is present if two protons are less than $5 \AA$ apart. In order to obtain this geometrical information one must know what diagonal position belongs to which proton. Several other kinds of spectra are used to facilitate the mapping of peaks to protons (the interpretation), and an example is the COSY spectrum in which cross peaks can appear between two diagonal resonance positions if the corresponding protons are separated by two or three chemical bonds. When all resonances in a NOESY spectrum are assigned to specific protons in the chemical structure, distance constraints can be set up and these can be used to construct a three-dimensional model of the protein, e.g. using the distance constraint algorithm [18]. The interpretation of the spectra, however, is a tedious task that can take months. It is clear that computer methods could greatly facilitate the task of the expert, if not by completely solving the spectra, then by interpreting large parts of them so that the expert can concentrate on the remainder.

The expert system described below is a reimplementation in $\mathrm{KEE}{ }^{\star}$ of an expert system named CINS, that was developed earlier in our department in collaboration with the department of Biophysical Chemistry of the University of Nijmegen. This expert system serves as a means of testing the refinement approach in classification expert systems in analytical chemistry. For the NMR interpretation problem, several 'brute force' methods have been reported [19-22]. The expert system described in this article uses essentially the same approach as the program described in ref. 19, but

\footnotetext{
$\overline{\text { Trademark }}$ of Intellicorp, Inc.
} 
additional knowledge is used to limit the number of possibilities in the search tree.

\section{The interpretation of NMR spectra}

The approach used is known as the "sequential assignment' strategy [17]. Its input consists of peak positions in seven types of NMR spectra, COSY (both in $\mathrm{H}_{2} \mathrm{O}$ and in $\mathrm{D}_{2} \mathrm{O}$ ), NOESY (idem), RCT (idem) and DQSY spectra. No information on peak shapes and intensities is used, and peak positions are used with an uncertainty of 0.01 ppm. The amino acid sequence is also known. The result of the interpretation gives the assignment of each peak in the NMR spectrum, i.e. a mapping of each proton in the chemical structure to a resonance position in the spectrum.

The first step in the spectrum interpretation comprises the identification of sets of peaks, belonging to protons in the same amino acid. Such a sel of peaks will hereafter be denoted as 'pattern'. As an example, the pattern belonging to the threonine depicted in Fig. 2 is (schematically) represented in Fig. 3. A simplified version of the internal computer representation of the pattern in Fig. 3 is given in Table 1.<smiles>CCCCCC(O)C(CCCC)C(C)=O</smiles>

Fig. 2. An example of an amino acid, in this case a threonine. Carbon atoms are indexed with greek letters, and the protons attached to them will be indicated likewise in the text.

In the second step of the spectrum interpretation each pattern found in the first part is classified as belonging to a type of amino acid, sometimes a specific type like glycine, sometimes a group of amino acids, like aromatic amino acids. Finally, data from NOESY spectra are used, together with the results of the previous assignment part, to establish a list of all patterns that are possible neighbours in the sequence. So, for a combination of two patterns to be valid for e.g. the amino acid combination valine alanine, the first pattern should have positive support for being a valine; likewise the second for alanine. Additionally, NOE cross peaks between the two patterns should be present to make sure the patterns can be neighbours. These pairs of neighbours are

\section{TABLE 1}

The computer representation of the threonine pattern in Fig. 3

\begin{tabular}{ll}
\hline PATTFRN-1 & \\
\hline ALPHA-BETA-PEAK(S) & $(4.554 .07)$ \\
VALUES & The cross peak(s) between the $\alpha$ and $\beta$ resonance positions \\
COMMENT & $(4.551 .4)$ \\
ALPHA-GAMMA & The cross peak(s) between the $\alpha$ and $\gamma$ resonance positions \\
VALUES & $(4.071 .4)$ \\
COMMENT & The cross peak(s) between the $\beta$ and $\gamma$ resonance positions \\
BETA-GAMMA-PEAK(S) & $(8.744 .07)$ \\
VALUES & The cross peak(s) between the amide and $\beta$ resonance positions \\
COMMENT & $(8.741 .4)$ \\
N-BETA & The cross peak(s) between the amide and $\gamma$ resonance positions \\
VALUES & $(8.744 .55)$ \\
COMMENT & The cross peak between the amide and $\alpha$ resonance positions \\
VALAMMA & COMMS
\end{tabular}




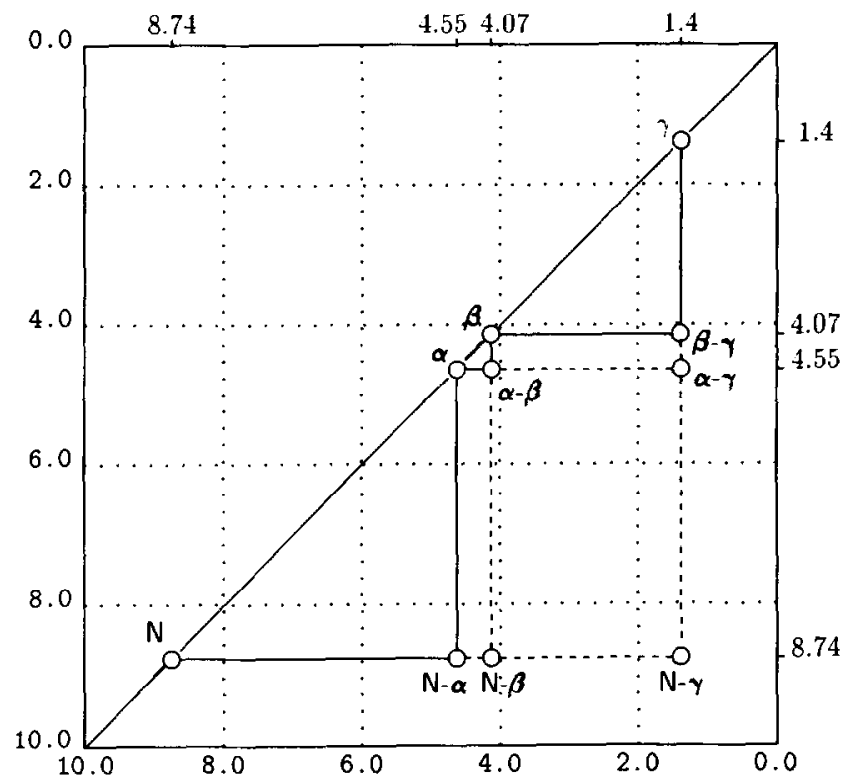

Fig. 3. The pattern of threonine-11 in the protein BPTI (see text). The letters in the pattern indicate the resonance positions of the protons in the amino acid of Fig. 2. Part of the computer representation of the pattern depicted here is shown in Table 1.

then chained to each other to obtain a complete sequence of patterns that can be mapped onto the amino acid sequence. As there are in most cases a lot of pattern candidates for separate amino acids, the search tree grows very rapidly in the beginning, but as the chain grows possibilities start to exclude each other because of the fact that a pattern may only appear once in a chain.

\section{The implementation of the pattern search expert system}

In this section we discuss the implementation of the first part of the expert system, in which patterns are found in the spectra; the subsequent part, in which the patterns are mapped to amino acids, has not yet been implemented. The input of this part of the expert system consists of the positions of the peaks in the NMR spectra, taken from the literature. The output consists of a list of patterns discovered, where each pattern contains resonance positions of protons in one amino acid. After the expert system is finished, the refinement module is activated automatically and compares the patterns found by the expert system with the patterns found by the human expert (i.e. the original articles cited). The latter are stored in a separate database. Refinements are then proposed by the system, if necessary, and after incorporation of these refinements a new consultation can begin with, one hopes, a better performance. At the end of a refining session several rounds of training have been performed, and the expert system should be able to reproduce the patterns found by human experts as closely as possible. The refinement strategy will be explained in greater detail below.

First of all, a list of so-called start peaks is set up; this list contains all peak positions that fall within the region of the spectrum that normally contains resonances due to interactions between the amide and $\mathrm{C}_{\alpha}$ protons. These start peaks serve as the starting point from which the remainder of the individual patterns are sought. It is of course possible that a peak in the start peak region is not an amide- $\mathrm{C}_{\alpha}$ cross peak; the pattern derived from such a start peak is then of no significance but will be taken into account because there is no way of telling beforehand which peaks in the start peak region are genuine start peaks and which are not. It is also possible that no start peak can be found belonging to an amino acid (as is the case for proline, for instance, since this amino acid does not have an amide proton); in that case no pattern will be found which can be mapped to that amino acid. After the patterns are initialized all peaks that have one coordinate in common with the $\alpha$-position of the start peak are considered as possible $\beta$-peaks. The amount of support for each of these possible $\alpha$ - $\beta$-peaks is then determined. This can be done in a number of ways, and in the past numerical values were often used to indicate the level of support of (or belief in) a hypothesis (i.e. a $\beta$-position). In our case linguistic entities were used, like 'major', 'medium' and 'minor', since these are easier to estimate than numerical certainty factors. If, for example, a cross peaks is found between a possible $\beta$-position and the amide position of the start peak, this is considered to be a major piece of support that the $\beta$-position is correct. If a cross peak is found between the $\alpha$-position and twice the value of another $\beta$-position in the DQSY spectrum, this is considered 


\section{TABLE 2}

The default types of evidence in the $\alpha-\beta$ and $\beta-\gamma$ search

The two leftmost columns contain the cross peaks that can be considered as evidence in the $\alpha-\beta$ search; the second column gives the relative importance of finding the given cross peak in a spectrum. Cross peaks $\alpha-\beta_{1}+\beta_{2}$ and $\alpha-2 * \beta$ are found in DQSY spectra (see ref. 17). The cross peaks looked for in the $\beta-\gamma$ search are gathered in the two rightmost columns. The same evidence may be found in more than one spectrum and may then be considered to have different importance. See, e.g. $\alpha-\gamma$; this peak can be found in RCT and in NOESY spectra, but because of the much larger number of peaks in the latter, finding a peak in that spectrum is considered to be of less importance.

\begin{tabular}{lllll}
\hline$\alpha-\beta$ search & & & $\beta-\gamma$ search & \\
\cline { 1 - 1 } Cross peaks & Importance & & Cross peaks & Importance \\
\hline $\mathrm{N}-\beta$ & major & $\alpha-\gamma$ (in RCT) & major \\
$\alpha-\beta_{1}+\beta_{2}$ & medium & $\alpha-\gamma$ (in NOESY) & medium \\
$\alpha-2 * \beta$ & medium & $\mathrm{N}-\gamma$ & medium \\
$\beta_{1}-\beta_{2}$ & minor & $\gamma_{1}-\gamma_{2}$ & minor \\
\hline
\end{tabular}

only of medium importance, and the former peak position will be preferred above the latter for the $\beta$ peak in the pattern. Thus, different cross peaks support a hypothesis (a peak position for the $\beta$ proton) to a different extent. Supporting cross peaks, together with their relative importances, are gathered in Table 2. According to the quantity of support each $\beta$-candidate has collected, the candidates are classified into the classes PROBABLES, POSSIBLES, IMPROBABLES and IMPOSSIBLES. Rules that decide in what class a candidate is classified use three thresholds: 'major-limit', 'medium-limit' and 'minor-limit'. For example if the number of major supports of a candidate is larger than the 'major-limit', a peak is classified as PROBABLE, regardless of the other support it has gathered. For each pattern the $\beta$-candidate(s) with the best classification is (are) selected. In Fig. 4 we give an example in which there are three candidates for a $\beta$ position; searching from the $\alpha$ position (4.55) three cross peaks are found: (4.55 4.07), (4.55 3.11) and (4.55 2.98). The best one (4.07) is classified as 'PROBABLE' because of the amount of support it has gathered (one major and one medium); the other two candidates are classified as 'IMPROBABLE' (only one piece of 'minor' importance) and are rejected, because a better alternative is at hand. It may be that an incorrect $\beta$ peak has, by chance, gathered more support than the correct one, either because some supporting cross peaks are absent in the spectrum or because cross peaks are found at positions that are thought to support the incorrect candidates. Overlapping patterns occur when two patterns share the same $\beta$ resonance position, for example, and then the expert system will probably also find identical $\gamma$-positions, while this is very likely to be untrue. This kind of error cannot be prevented, and therefore a $100 \%$ performance is not possible.

After this search for $\alpha-\beta$ peaks the search for $\gamma$-methyl protons can proceed in the same way, starting from the $\beta$-position(s) found in the previous step. No attempt is made to find other $\gamma$-peaks or $\delta$ - and e-peaks because of the crowded regions in the spectra in which they normally appear. Different types of support can also be gathered in the search for $\beta-\gamma$ peaks; they, too, are collected in Table 2. Now the pattern search part of the expert system is finished and the patterns discovered can be assigned to types of amino acids.

In the remainder of this article we will discuss the effects of the refinement approach on the performance of the pattern search part of the

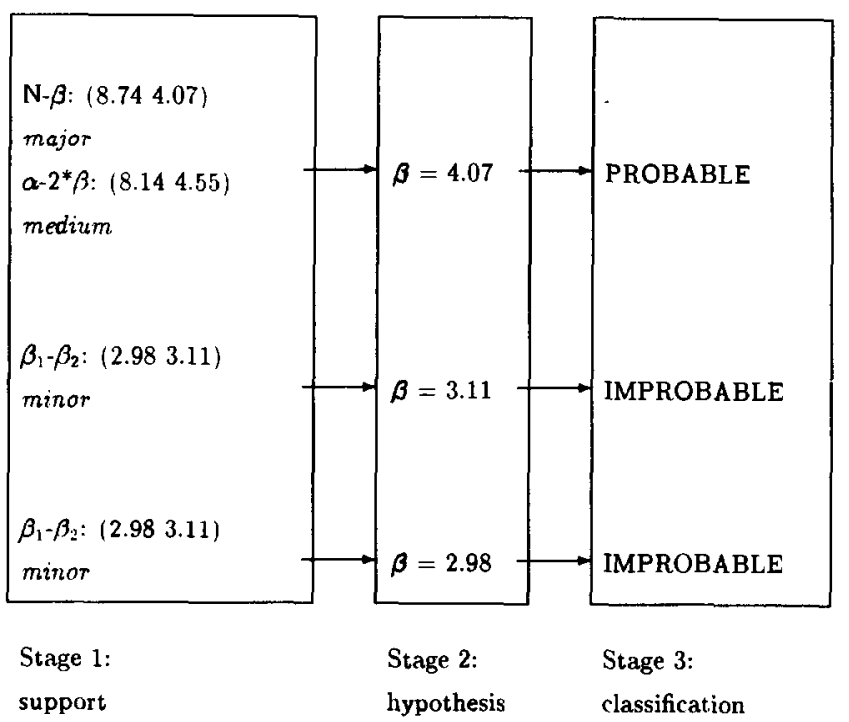

Fig. 4. Classification of candidates for the $\beta$-position, starting from start peak (8.74 4.55). Only $\beta=4.07$ will be selected. 
Refinable limits in the patternsearch expert system:

SPECIALIZABLE LIMITS:

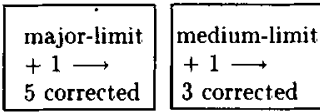

GENERALIZABLE LIMITS

\begin{tabular}{|c|c|c|}
\hline major-lin & medium-limit & minor-limit \\
\hline$-1 \longrightarrow$ & $-2 \longrightarrow$ & $-1 \longrightarrow$ \\
\hline 4 corrected & 4 corrected & 8 corrected \\
\hline
\end{tabular}

\begin{tabular}{|l|l|l|l|l|l|l|l|l}
\hline $\begin{array}{l}\text { CONTINUE } \\
\text { CHAINING }\end{array}$ & DONE \\
\hline
\end{tabular}

Fig. 5. The refinement screen.

NMR expert system. The results published in the literature are stored in a separate database and the results of the expert system are compared with them. As only the pattern search part will be discussed here in relation to refinements, only peak positions in found patterns have to be compared with peak positions in assigned amino acids. Only incorrect $\alpha-\beta$ and $\beta-\gamma$ peaks are considered to be FP or $\mathrm{FN}$; other peaks are supporting peaks and should therefore always be present if the correct $\alpha-\beta$ and $\beta-\gamma$ peaks are found. The refinable parameters in the expert system described above are the importances of the individual supporting cross peaks and the thresholds (major-, medium- and minor-limit) that decide in what class a $\beta$ - or $\gamma$-candidate is classified (PROBABLE, POSSIBLE, IMPROBABLE or IMPOSSIBLE). By modifying the above parameters the results of the expert system can be changed, so that an optimal setting can be found.

In Fig. 5 we give an example of the screen that is shown to the user after validation of the results and calculation of the proposed refinements. The small boxes in the center give the suggestions of the refinement system for changes to be made in the knowledge base. If the user agrees to one or more of these refinements, clicking in one of the small boxes in the center will cause the given refinement to be incorporated in the knowledge base. For example, clicking in the upper left box causes the major-limit to be raised by 1 , and five incorrect cases may be corrected by this refinement. Lowering the major-limit by 1 , however, has a chance of correcting four other misinterpreted cases. Combinations of refinements may also be selected; in that case another box appears indicating the effect of the combined refinement. After all refinements are incorporated, clicking in the 'DONE' box causes the system to consult the refined rules again. The estimated numbers of corrected cases are based on the cases in which the given limit is responsible for the misinterpreted cases; the actual number of corrected cases will be lower because previously correct cases may become incorrect because of the refinement, and also other limits may be responsible for the misinterpreted case. The numbers thus represent upper bounds of the numbers of corrected cases after refinements.

\section{RESULTS AND DISCUSSION}

In this section we discuss the results of the NMR expert system and the effect of the refinement strategy on its performance. Three test cases are considered: the spectra of the proteins BPTI (Bovine Pancreatic Trypsine Inhibitor), E-L30, and Tendamistat. The spectra of E-L30 were measured at the laboratory of Biophysical Chemistry of the University of Nijmegen [23,24]; the data of BPTI and Tendamistat were adapted from the literature $[25,26]$ (see ref. 19 for more details).

\section{Refinement results for protein BPTI}

The protein BPTI consists of 58 amino acids, and the NMR spectra contain 56 start peaks. Six amino acids do not have start peaks (the $\mathrm{N}$ terminus, four prolines and a glycine), whereas four glycines have two start peaks. Thus, 52 correct patterns should be found by the expert system; if more patterns are found the incorrect ones 


\section{TABLE 3}

Refinement session for BPTI

In case of the proteins E-L30 and Tendamistat, the best results in the $\beta-\gamma$ search are obtained with major-, medium-, and minor-limits of 1,2 and 2 , respectively.

\begin{tabular}{lcccc}
\hline \multicolumn{3}{l}{ Major-limit Medium-limit Minor-limit Weights } & No. incorrects \\
\hline$\alpha-\beta$ search & 1 & 1 & default & 5 \\
1 & 1 & 2 & default & 3 \\
1 & 2 & 1 & default & 5 \\
1 & 2 & 1 & $\beta_{1}-\beta_{2}$ & 2 \\
1 & \multicolumn{3}{c}{ medium } \\
$\beta$ - $\gamma$ search & 1 & 1 & default & 4 \\
1 & 2 & 2 & default & 4 \\
1 & 1 & 2 & default & 3 \\
2 & &
\end{tabular}

will be filtered out in the assignment phase. 'Correct' in this case means the finding of all $\alpha-\beta$ and $\beta-\gamma$ peaks given in the literature, together with the support they have gathered. With the original settings of the refinable parameters mentioned above, as given by the expert, the expert system deduced 43 of the 52 patterns correctly, and after refinements this increased to 47 out of 52 patterns. These figures correspond to performances of $83 \%$ and $90 \%$, respectively. Table 3 presents an example of a refinement run in which the expert tries several settings, until the best one is found.

\section{Refinement results for protein $E-L 30$}

E-L30 is a ribosomal protein from $E$. coli, containing 58 amino acids. For six of the 58 amino acids no start peak could be found while two glycines gave rise to two start peaks. This yielded 54 start peaks, corresponding with 52 amino acids in the sequence. The expert system found 41 correct patterns, a performance of $79 \%$. After refinement, 2 additional patterns were deduced correctly, a performance improvement of 4\%. The optimal settings differed slightly from the ones found in the case of BPTI. We will return to this in the Discussion section, below.

\section{Refinement results for protein Tendamistat}

Tendamistat is a larger protein than the previous two, consisting of 74 amino acids. Three pro- lines do not have start peaks and seven glycines have two start peaks. Thus, 71 patterns should be mapped to amino acids. Before refinement 40 out of 71 patterns were found correctly (56\%); after refinement this was increased to 45 patterns, a performance of $64 \%$. This is significantly lower than the performance in the other two proteins because of extensively overlapping patterns. In most cases, however, this leads to FP peaks, which in the following assignment parts should be filtered out. The figure of $64 \%$ thus represents a very pessimistic view of the performance of the expert system. The optimal settings were equal to the ones found in the case of E-L30.

\section{Discussion}

The overall performance of the expert system is comparable to the performance of a human expert, even before refinements; because of the extensive feedback from the next steps in the interpretation (global and sequential assignments of patterns to amino acids), the eventual performance will be much higher. It should also be noted that an incorrect result does not mean that the next steps in the interpretation are bound to fail. It may very well be that a pattern with, e.g., an extra $\alpha-\beta$ peak will be classified correctly and can be incorporated in the sequence of patterns at just the right place. Only one peak will then be interpreted falsely instead of a whole set of peaks. After this assignment phase the patterns will be checked if they are still consistent (at that stage, a peak may in principle appear only once in all patterns), and errors due to the pattern search part may be filtered out.

The results of the three proteins given in the above sections indicate that the refinement approach can help to improve expert system performance, even in a relatively simple system as the one described above. In the proteins E-L30, BPTI and Tendamistat performance improvements of 4 , 9 and $8 \%$ are reached, respectively, where it should be noted that the knowledge used in the initial implementation was already extensively tested in the expert system CINS mentioned earlier. In Table 4 the results for the three proteins are gathered using the thresholds and weights of sup- 
TABLE 4

Results for the three proteins

\begin{tabular}{|c|c|c|c|c|c|}
\hline \multirow[t]{2}{*}{ Protein } & \multirow[t]{2}{*}{ No. of amino acids } & \multicolumn{2}{|c|}{$\begin{array}{l}\text { Incorrect } \\
\text { patterns } \\
\text { (initial) }\end{array}$} & \multicolumn{2}{|c|}{$\begin{array}{l}\text { Incorrect } \\
\text { patterns } \\
\text { (optimal) }\end{array}$} \\
\hline & & No. & 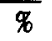 & No. & $\%$ \\
\hline E-L30 & 52 & 11 & 21 & 9 & 17 \\
\hline BPTI & 52 & 9 & 17 & 6 & 12 \\
\hline Tendamistat & 71 & 31 & 44 & 26 & 37 \\
\hline Total & 175 & 51 & 29 & 41 & 23 \\
\hline
\end{tabular}

TABLE 5

Final and optimal settings for refinable parameters

The weights of the individual pieces of evidence in the initial configuration are given in Table 2 . In the optimal configuration, the weight of the $\beta_{1}-\beta_{2}$ supporting cross peak has been changed to medium.

\begin{tabular}{|c|c|c|c|c|c|}
\hline \multicolumn{3}{|l|}{$\alpha-\beta$ parameters } & \multicolumn{3}{|l|}{$\beta-\gamma$ parameters } \\
\hline Parameter & Initial & Optimal & Parameter & Initial & Optimal \\
\hline$\overline{\text { Major-limit }}$ & 1 & 1 & Major-limit & 1 & 1 \\
\hline Medium-limit & 1 & 2 & Medium-limit & 1 & 2 \\
\hline Minor-limit & 1 & 1 & Minor-limit & 1 & 2 \\
\hline
\end{tabular}

ports that are considered best. These optimal settings are gathered in Table 5, together with the settings originally given by the expert. In different trainings sets (in this case each protein was used as a separate trainings set) different settings may lead to optimal results, but then some kind of compromise will have to be found. In this case, the 'optimal' settings found for E-L30 and Tendamistat were equal, but the BPTI settings differed somewhat. Using the E-L30/Tendamistat optimal settings resulted in one extra misinterpreted case in BPTI, which is considered to be acceptable.

\section{CONCLUSIONS AND OTHER APPLICATIONS}

We have demonstrated an application of the refinement approach in analytical chemistry. It appeared that the test expert system for the interpretation of two-dimensional NMR spectra of proteins yielded better results after several rounds of training using the aforementioned approach. With larger knowledge bases in particular it is likely that the gain of such an approach will be considerable. Other aspects deserve attention, too. In the case of the NMR expert system more or less ideal spectra were used, read from the literature. If the expert system were to work for experimental spectra, it is very well possible that other values for certain parameters will yield better results. The refinement approach gives an opportunity to smoothly adjust the knowledge base to the new and more difficult situation. In other fields, too, where it may be hard to find experimental data-sets the expert system may initially be trained using an available set, followed by fine-tuning during the field work. The flexibility thus obtained may also be used to apply expert systems to problems for which they were not originally designed: as an example, it may be possible to rewrite, with a minimum of effort, an expert system that is meant to classify a specific class of chemicals so that it also is able to classify a different (but related) class of chemicals. This advantage of the refinement approach may very well become an even more important aspect than the performance improvements obtained, and research is going on in our department on this subject. Another advantage of the above approach is that internal consistency can be maintained and if a new piece of evidence or a new rule is added, then the knowledge base can easily be trained to adjust to the new situation. Finally, the considerable debugging capacities of using the refinement approach in the implementation phase of an expert system must be mentioned.

\section{REFERENCES}

1 D. Goulder, T. Blaffert, A. Blokland, L. Buydens, A. Chhabra, A. Cleland, N. Dunand, H. Hindriks, G. Kateman, H. van Leeuwen, D. Massart, M. Mulholland, G. Musch, P. Naish, A. Peeters, G. Postma, P. Schoenmakers, M. de Smet, B. Vandeginste and J. Vink, Expert systems for chemical analysis, Chromatographia, 26 (1988) 237-243.

2 B.A. Hohne and T.H. Pierce (Editors), Expert System Applications in Chemistry, American Chemical Society, Washington, DC, 1989.

3 R.E. Dessy, Expert systems I, Analytical Chemistry, 56 (1984) 1200A-1212A. 
4 R.E. Dessy, Expert systems II, Analytical Chemistry, 56 (1984) 1312A-1332A.

S L. Land and T. Mulhall, Developing cooperative knowledge-based systems, in N. Schadbolt (Editor), Proceedings on Expert Systems VI, Landon, 1989, Cambridge University Press, Cambridge, 1989, pp. 90-103.

6 C.M. Kitto and J.H. Boose, Selecting knowledge acquisition tools and strategies based on application characteristics, International Journal of Man-Machine Studies, 31 (1989) 149-160.

7 T.M. Mullin, Experts' estimation of uncertain quantities and its implications for knowledge acquisition, IEEE Transactions on Systems, Man and Cybernetics, 19 (1989) 616-625.

8 B.J. Cragun and H.J. Steudel, A decision-table based processor for checking completeness and consistency in rule-based expert systems, International Journal of ManMachine Studies, 26 (1987) 633-648.

9 T.A. Nguyen, W.A. Perkins, T.J. Laffey and D. Pecora, Checking an expert systems knowledge base for consistency and completeness, in Proceedings of the International Journal Conference on Artificial Intelligence, Los Angeles, CA, Morgan Kaufman, San Mateo, CA, 1985, pp. 375-378.

10 D.L. Nazareth, Issues in the verification of knowledge in rule-based systems, International Journal of Man-Machine Studies, 30 (1989) 255-271.

11 P. Politakis and S.M. Weiss, Using empirical analysis to refine expert systems knowledge bases, Artificial Intelligence, 22 (1984) 23-48.

12 A. Ginsberg, S.M. Weiss and P. Politakis, Automatic knowledge base refinement for classification systems, Artificial Intelligence, 35 (1988) 197-226.

13 G.J. Klir and T.A. Folger, Fuzzy Sets, Uncertainty, and Information, Prentice Hall, Englewood Cliffs, NJ, 1988.

14 S.J. Henkind and M.C. Harrison, An analysis of four uncertainty calculi, IEEE Transactions on Systems, Man and Cybernetics, 17 (1988) 700-714.

$15 \mathrm{~T}$. Whalen and B. Schott, Alternative logics for approximate reasoning in expert systems: a comparative study, International Journal of Man-Machine Studies, 22 (1985) 327-346.
16 T.R. Gruber and P.R. Cohen, Design for acquisition: principles of knowledge-system design to facilitate knowledge acquisition, International Journal of Man - Machine Studies, 26 (1987) 143-159.

17 K. Wüthrich, NMR of Proteins and Nucleic Acids, Wiley, New York, 1986.

18 G.M. Crippen, Distance Geometry and Conformational Calculations, Research Studies Press, Chichester, 1981.

19 F.J.M van de Ven, PROSPECT, a program for automated interpretation of 2D NMR spectra, Journal of Magnetic Resonance, 86 (1990) 633-644.

20 C. Cieslar, G.M. Clore and A.M. Gronenborn, Computeraided sequential assignment of protein ${ }^{1} \mathrm{H}$ NMR spectra, Journal of Magnetic Resonance, 80 (1988) 119-127.

21 C.D. Eads and I.D. Kunz, Program for computer-assisted sequential assignment of proteins, Journal of Magnetic Resonance, 82 (1989) 467-482.

22 P.L. Weber, J.A. Malikayil and L. Mueller, Automated elucidation of $J$ connectivities in ${ }^{1} \mathrm{H}$ NMR spectra, Journal of Magnetic Resonance, 82 (1989) 419-426.

23 F.J.M. van de Ven and C.W. Hilbers, Residue-specific assignments of resonances in the ${ }^{1} \mathrm{H}$ nuclear magnetic resonance spectrum of ribosomal protein E-L30 by systematic application of two-dimensional Fourier transform nuclear magnetic resonance methods, Journal of Molecular Biology, 192 (1986) 389-417.

24 F.J.M. van de Ven and C.W. Hilbers, Sequential resonance assignments as a basis for the determination of a three-dimensional structure of protein E-L30 of Escherichia coli, Journal of Molecular Biology, 192 (1986) 419-441.

$25 \mathrm{G}$. Wagner and $\mathrm{K}$. Wüthrich, Sequential resonance assignments in protein ${ }^{1} \mathrm{H}$ nuclear magnetic resonance spectra. Basic pancreatic trypsin inhibitor, Journal of Molecular Biology, 155 (1982) 347-366.

26 A.D. Kline and K. Wüthrich, Complete sequence-specific ${ }^{1} \mathrm{H}$ nuclear magnetic resonance assignments for the $\alpha$ amylase polypeptide inhibitor Tendamistat from Streptomyces tendae, Journal of Molecular Biology, 192 (1986) 869-890. 